\title{
Antibiorésistance Et Facteurs De Virulence Des Souches D'escherichia Coli Isolées Des Carcasses Bovines Du Bénin
}

\section{Hélène Ahouandjinou, M.Sc. \\ Farid Baba-Moussa, PhD}

Laboratoire de Microbiologie et de Technologie Alimentaire, Faculté des Sciences et Techniques/Université d’Abomey-Calavi,

ISBA-Champ de foire Cotonou, BENIN.

\section{Gbaguidi Bertin, PhD}

Ecole Polytechnique d'Abomey-Calavi, (EPAC) - Bénin.

\section{Haziz Sina, PhD}

Laboratoire de Biologie et de Typage Moléculaire en Microbiologie, Faculté des Sciences et Techniques/Université d'Abomey-Calavi, Cotonou, BENIN.

\section{Kifouli Adéoti, PhD}

Laboratoire de Microbiologie et de Technologie Alimentaire,

Faculté des Sciences et Techniques/Université d'Abomey-Calavi,

ISBA-Champ de foire Cotonou, BENIN.

Wassiyath Mousse, PhD

Sylviane Pouadjeu-Wouansi, B.Sc.

Laboratoire de Biologie et de Typage Moléculaire en Microbiologie,

Faculté des Sciences et Techniques/Université d'Abomey-Calavi, Cotonou, BENIN.

\section{Fatiou Toukourou, PhD}

Laboratoire de Microbiologie et de Technologie Alimentaire,

Faculté des Sciences et Techniques/Université d'Abomey-Calavi,

ISBA-Champ de foire Cotonou, BENIN.

\section{Mohamed Soumanou, PhD}

Laboratoire d'Etude et de Recherche en Chimie Appliquée de l'Ecole Polytechnique d'Abomey-Calavi, Cotonou, BENIN

\section{Lamine Baba-Moussa, PhD}

Laboratoire de Biologie et de Typage Moléculaire en Microbiologie,

Faculté des Sciences et Techniques/Université d'Abomey-Calavi, Cotonou, BENIN 


\section{Abstract}

The meat processing conditions expose it to several contaminations including the microbial. The present study was designed to assess the antibiotic resistance and toxin production by Escherichia coli strains isolated bovine carcasses collected in the slaughterhouse of Cotonou / Porto-Novo in Benin. Thus, a total of 240 samples was collected from 60 beef carcasses by the destructive method. The E. coli strains were identified by conventional microbiological and biochemical methods. The susceptibility of strains to 15 antibiotics was assessed by disc diffusion method on agar medium. The phenotypic identification of strains producing penicillinase and BSLE was performed respectively by the tubes acidimetric test and the double halo method. PCR was used to detect genes encoding the toxins and $\beta$-lactamases. The results showed that $57.92 \%$ of the samples were contaminated with $E$. coli, with highest rate recorded in the samples collected from arm. The susceptibility to 15 antibiotics tested has shown that all the isolated strains were multi-resistant with a high proportion to ceftriaxone (88.49\%). the lowest resistance rate $(\sim 1 \%)$ was recorded with gentamicin. The E. coli strains producing $\beta$-lactamase carried multidrug resistance genes blaSHV (26.92\%) and blaTEM (40.29\%). To end, our data revealed the presence of EHEC pathovar (12.82\%), responsible for the "traveler" disease. Thus, meat coming from slaughterhouses are therefore a potential source of food poisoning.

Keywords: Bovine carcasses, E. coli, antibiotics, $\beta$ lactamase, Bénin

\section{Résumé}

Les conditions de traitement de la viande l'exposent à des contaminations de tout genre dont celle microbienne. La présente étude a eu pour but d’évaluer la résistance aux antibiotiques et la production de toxines par les souches d'Escherichia coli isolées des carcasses bovines de l'abattoir Cotonou/Porto-Novo au Bénin. Pour ce faire, un total de 240 échantillons a été collecté sur 60 carcasses bovines par la méthode destructive. Les souches $\mathrm{d}^{`} E$. coli ont été identifiées par les méthodes microbiologiques et biochimiques conventionnelles. La susceptibilité des souches aux antibiotiques a été évaluée par la méthode de diffusion des disques sur milieu gélosé. L’identification phénotypique des souches productrices de la pénicillinase et de BSLE a été faite respectivement par le test d'acidimétrique en tubes et la méthode du doublehalo. La PCR a permis de rechercher les gènes codant pour les toxines et les $\beta$-lactamases. Les résultats ont révélé que 57,92\% des échantillons analysés étaient contaminés par $E$. coli, avec un taux beaucoup plus élevé (70\%) au niveau du bras. La 
susceptibilité des souches aux 15 antibiotiques testés montre que toutes les souches étaient multi-résistantes avec une forte proportion à la ceftriaxone (88,49\%). Par contre, une faible résistantes à la gentamicine $(1,44 \%)$ était observée. Les souches $E$. coli productrices de $\beta$ lactamase étaient porteuses des gènes de multi-résistance bla ${ }_{\mathrm{SHV}}(26,92 \%)$ et bla $\mathrm{TEM}_{\mathrm{TEM}}(40,29 \%)$. Enfin, nos données revèlent la présence du pathovar EHEC (12,82\%), responsable de la maladie du "voyageur ». Les viandes sorties des abattoirs sont donc de potentielle source d'intoxications alimentaires.

Mots clés : Carcasses bovines, E. coli, antibiotiques, $\beta$ lactamase, Bénin.

\section{Introduction:}

La préservation de la santé constitue l'une des préoccupations majeures de tous les temps, étant donné sa place primordiale dans tout processus de développement. L’amélioration de la santé humaine repose sur plusieurs stratégies à l'instar de la consommation des aliments sains. La viande est une denrée hautement périssable dont la qualité hygiénique dépend de la contamination pendant les opérations d'abattage et de découpe. De plus, il y a un risque de développement et de croissance des flores contaminantes pendant le refroidissement, le stockage et la distribution (El-Hadef et al., 2005). Dans les pays développés, près de $30 \%$ de la population contracte une affection d'origine alimentaire et la proportion est sans aucun doute plus élevée encore dans les pays en développement (WHO, 2011). L’une des bactéries les plus incriminées dans les cas d'infection alimentaire demeure Escherichia coli.

En effet, malgré le fait que cette bactérie soit symbiotiquement retrouvée dans l'intestin des êtres humain, il existe certaines souches qui sont nuisibles et créent des infections diverses (Kaper et al. 2004). Ainsi, plusieurs études se sont penchées sur la pathogénicité de certaines souches d'E. coli. De ce fait, il ressort que $E$. coli est un agent pathogène majeur responsable des pathologies infectieuses, aussi bien en milieu hospitalier qu'en milieu extrahospitalier (Johnson et Russo, 2005). La pathogénicité dans ce cas peut se traduire par la sécrétion des enzymes et toxines qui ont un effet cytopathogène sur les cellules humaines (Clifton-Hadley, 2000). Pour la lutte contre ces microorganismes, les antibiotiques sont couramment utilisés (De Wals, 2007). Mais, depuis un certain temps, les bactéries ont commencé par acquérir des résistances à plusieurs antibiotiques simultanément entrainant des échecs thérapeutiques.

Il est donc très important de connaitre le comportement des souches bactériennes en fonction de leur provenance. C'est dans ce cadre que se situe notre présent travail dont l'objectif était non seulement de dresser le profil de résistance des souches d'E. coli isolées des carcasses bovines destinées à la 
consommation humaine mais aussi de rechercher certains des facteurs de virulence que ces souches isolées peuvent produire.

\section{Matériel et Méthodes:}

\section{Echantillonnage et collecte des échantillons}

Les échantillons ont été collectés en utilisant la méthode par excision sur une période de cinq (5) mois allant du vingt-sept juillet au vingt-huit décembre 2015. Les prélèvements d’au moins $5 \mathrm{~cm}^{2}$ ont été effectués de manière aléatoire sur une journée de la semaine, sur 5 demi-carcasses issues de 5 carcasses différentes. D’une séance d’échantillonnage à l'autre, les prélèvements ont été réalisés, en alternance, sur 5 demi-carcasses gauches, puis 5 droites. Par jour de collecte, 20 échantillons ont été prélevés au niveau du bras (Br), du cou (Co), de la cuisse $(\mathrm{Cu})$ et du flanc $(\mathrm{Fl})$ de la carcasse choisie (Figure 1). Ainsi, un total de 240 échantillons ont été collectés étiquetés puis acheminés au laboratoire dans une glacière (entre $0^{\circ} \mathrm{C}$ et + $4^{\circ} \mathrm{C}$ ) pour les différentes analyses.

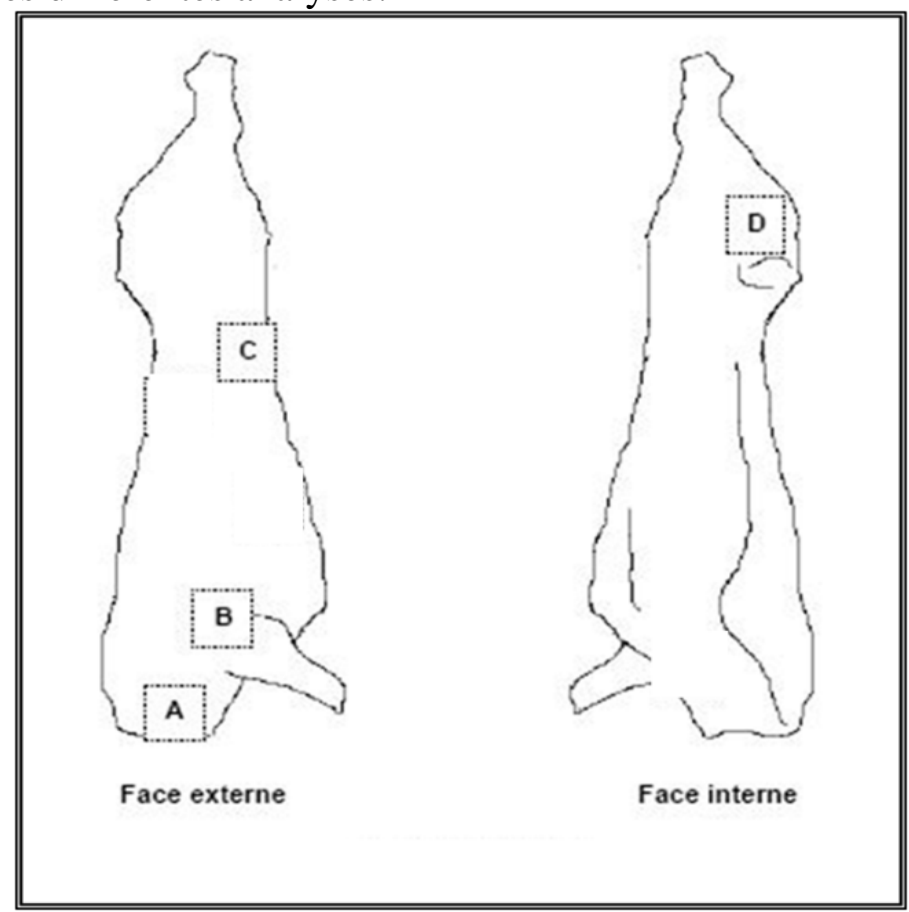

A : le cou ; B : le bras ; C : le flanc (la face externe du flanc) ; D : la cuisse (face interne de la cuisse)

Figure 1. Sites de prélèvement des échantillons de carcasses bovines analysés.

\section{Traitement des échantillons collectés}

\section{Isolement et Identification des souches de E. coli}

L’isolement et l’identification ont été réalisés par les méthodes classiques utilisées en microbiologie. Ainsi, les souches d'E .coli ont été 
isolées en utilisant la gélose TBX. La confirmation de l'espèce $E$. coli a été faite suite à la réalisation de la coloration de Gram et des tests complémentaires que sont : les tests de production d'indole, de catalase, de fermentation du glucose et lactose, de mobilité et d'utilisation du mannitol. Une fois identifiée, les souches ont été utilisée pour les tests de resistance aux antibiotiques, les productions de toxines et la recherche des gènes codant pour la production de toxines.

\section{Antibiorésistance des souches d'Escherichia coli aux antibiotiques}

L’étude de la sensibilité aux antibiotiques a été réalisée par la méthode de diffusion sur milieu gélosé Mueller-Hinton selon les normes et les recommandations du comité de l'antibiogramme de la société française de la microbiologie (SFM, 2015). Pour ce faire, quinze antibiotiques (Oxoid) ont été testés: Imipenème $(10 \mu \mathrm{g})$, gentamicine $(10 \mu \mathrm{g})$, fosfomycine (50 $\mu \mathrm{g})$, amoxicilline + acide clavuanique $(30 \mu g)$, ciprofloxacine $(5 \mu g)$, triméthoprime-sulfaméthoxazole $(25 \mu \mathrm{g})$, céfixime $(5 \mu \mathrm{g})$, ampicilline (10 $\mu g)$, céfoxitine $(30 \mu g)$, ceftazidime $(10 \mu g)$, ceftriaxone $(30 \mu g)$, acide nalidixique $(30 \mu g)$, amikacine $(30 \mu g)$, tobramycine $(10 \mu g)$, Chloramphénicol (30 $\mu \mathrm{g})$. La lecture des diamètres d’inhibition, après $24 \mathrm{~h}$ d'incubation à $37^{\circ} \mathrm{C}$, a permis l'interprétation des résultats selon les recommandations du CA-SFM (2015).

\section{Détection phénotypique de la pénicillinase et des betalactamases à spectre élargi (BLSE)}

La recherche des souches des E. coli productrice de la pénicillinase a été faite par la méthode en milieu liquide décrite par Jalier et al. (1988). Cette méthode a consisté à émulsionner deux jeunes colonies de 24 heures dans $500 \mu \mathrm{l}$ d'eau physiologique stérile. A ce mélange, $150 \mu \mathrm{l}$ du réactif à la benzyl-pénicilline (600 mg de benzyl-pénicilline +350 $\mu \mathrm{l}$ d'eau distillée stérile + $\mathrm{NaOH}(1 \mathrm{M})$ + Rouge de phénol) extemporanément préparé à $\mathrm{pH} 8 \mathrm{y}$ ont été ajoutés puis incubés à $37^{\circ} \mathrm{C}$ pendant $1 \mathrm{~h}$. Pour le contrôle, une souche de référence d'E. coli ATCC 25922 a servi de témoin. Après incubation, un virage de la couleur rouge pourpre initial à l'orangé (ou jaune) dans les tubes indique que la souche testée est productrice de pénicillinase.

En ce qui concerne la recherche de la production de $\beta$-lactamses à spectre élargi (BLSE), la méthode du double halo à été utilisée (Thomson et Sanders 1992). Avant le test proprement dit, une gélose $\mathrm{MH}$ a été inondée avec une suspension bactérienne de $10^{6}$ bactéries $/ \mathrm{ml}$ dans de l'eau physiologique (SFM, 2015). Une fois le préalable réalisé, un disque d'amoxicilline + acide clavulanique (AMC) a été déposé au centre, à équidistance (30 mm), entre la Céfotaxime (CTX) et la Ceftriaxone (CRO). Après incubation à $37^{\circ} \mathrm{C}$ pendant $18 \mathrm{~h}$, le résultat positif se traduit par une 
potentialisation de la zone d'inhibition (double halo) en forme de tirebouchon autour des disques de CTX, CRO, en présence de l'AMC, ceci résultant de la synergie des $\beta$-lactamines avec l'acide clavulanique (Allouch et al., 1995).

\section{Détection genotypique des souche de E. coli productrices de BLSE et de toxines}

Cette détection a été faite par la réaction de polymérisation en chaine (PCR, acronyme anglais de Polymerase Chain Reaction) sur l'ADN total extrait. L'ADN total a été extrait par la methode de chauffage des précultures bacteriennes dans le but d'éclater les bacteries (Mousse et al. 2016). Les gènes de caractérisation des BLSE (bla $a_{\mathrm{TEM}}$, bla $\mathrm{C}_{\mathrm{CTX}-\mathrm{M}}$ et bla $\mathrm{a}_{\mathrm{SHV}}$ ) et de production de toxines (Véro toxine et entéro-toxine thermolabile) ont été recherchés à partir des séquences des amorces spécifiques (tableau 1). Ainsi, pour cette étude les amorces (Forward et Reverse) précédemment utilisées

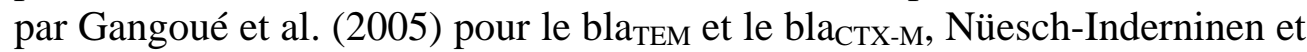
al. (1996) pour le blashv, Arranda et al. (2007) pour la Véro toxine et Gassama-Sow et al. (2004) pour l'entéro-toxine thermolabile, ont été utilisées.

Tableau 1: Séquences des amorces utilisées pour la recherche des E. coli productrices de BSLE et de toxines

\begin{tabular}{|c|c|c|c|c|}
\hline & Gènes & Amorces & Séquences des amorces (5’ 3 ?) & Taille (bp) \\
\hline \multirow{6}{*}{ : } & \multirow[b]{2}{*}{ bla $_{\text {TEM }}$} & OT-F & 5'-TTGGGTGCACGAGTGGGTTA-3’ & \multirow{2}{*}{467} \\
\hline & & OT-R & 5'-TAATTGTTGCCGGGAAGCTA-3' & \\
\hline & \multirow{2}{*}{ bla $_{\text {SHV }}$} & SHV-F & 5'-CGCCGGGTTATTCTTATTTGTCGC-3 & \multirow{2}{*}{1017} \\
\hline & & SHV-R & 5’-TCTTTCCGATGCCGCCGCCAGTCA-3 & \\
\hline & \multirow{2}{*}{ bla $_{\text {CTX-M }}$} & CTX-F & 5'-CGCTTTGCGATGTGCAG-3' & \multirow{2}{*}{550} \\
\hline & & CTX-R & 5’-ACCGCGATATCGTTGGT-3’ & \\
\hline \multirow{2}{*}{ EHEC } & \multirow{2}{*}{ VT } & VT-F & 5'-GAGCGAAATAATTTATATGTG-3' & \multirow{2}{*}{518} \\
\hline & & VT-R & 5'-TGATGATGGCAATTCAGTAT-3' & \\
\hline \multirow{2}{*}{ ETEC } & \multirow{2}{*}{ LT } & LT-F & 5’-GCGACAAATTATACCGTGCT-3’ & \multirow{2}{*}{315} \\
\hline & & LT-R & 5’-CCGAATTCTGTTATATATGT-3’ & \\
\hline
\end{tabular}

VT : vérotoxine ; LT : entérotoxine thermolabile ; EHEC : entérohemorrhagic E. coli ; ETEC : entérotoxigenic E.coli

La réaction de PCR était réalisée dans un milieu de $20 \mu$ constitué d'un master Mix 2x (10 $\mu$ l), d'amorces sens et anti-sens ( $1 \mu$ de chaque) et de 6 $\mu \mathrm{l}$ d'ADN. Les amplifications ont été réalisées dans un thermocycleur (3 PRIME BASE/02/ Serial : 30150 Made in UKA) selon le programme suivant : dénaturation intiale à $95^{\circ} \mathrm{C}(10 \mathrm{~min})$; 40 cycles constitués d'une dénaturation à $95^{\circ} \mathrm{C}(30 \mathrm{~s})$, d'hybridation à $50^{\circ} \mathrm{C}(30 \mathrm{~s})$ et d'élongation à $72^{\circ} \mathrm{C}(30 \mathrm{~s})$ et une élongation finale à $72^{\circ} \mathrm{C}$ pendant $10 \mathrm{~min}$. A la fin de la réaction, les produits PCR étaient maintenus à $4^{\circ} \mathrm{C}$ jusqu'à migration. 
La migration des produits issus de la PCR a été faite par électrophorèse (120 V, 45 min) sur gel d'agarose (1,5\%) additionné de BrET dans le TBE 1x. Un marqueur de $100 \mathrm{pb}$ (Promega, USA) a été utilisé comme marqueur de poids moléculaire. Après le temps de migration, les gels ont été visualisés sous lampe UV et la présence d'une bande fluorescente correspondant à la taille du fragment attendu signifie la présence du gène recherché.

\section{Analyse des données}

Les données recueillies ont été saisies dans une base de données Excel 2013. Quant aux différents tests statistiques ils ont été réalisés par des logiciels d'analyses (Stata, Epi info et Graph Pad Prism 5).

\section{Résultats}

\section{Contamination des carcasses bovines}

Sur les 240 échantillons de carcasse collectés, 139 étaient contaminés par E. coli, soit un taux de contamination de 57,92\% (Figure 2).

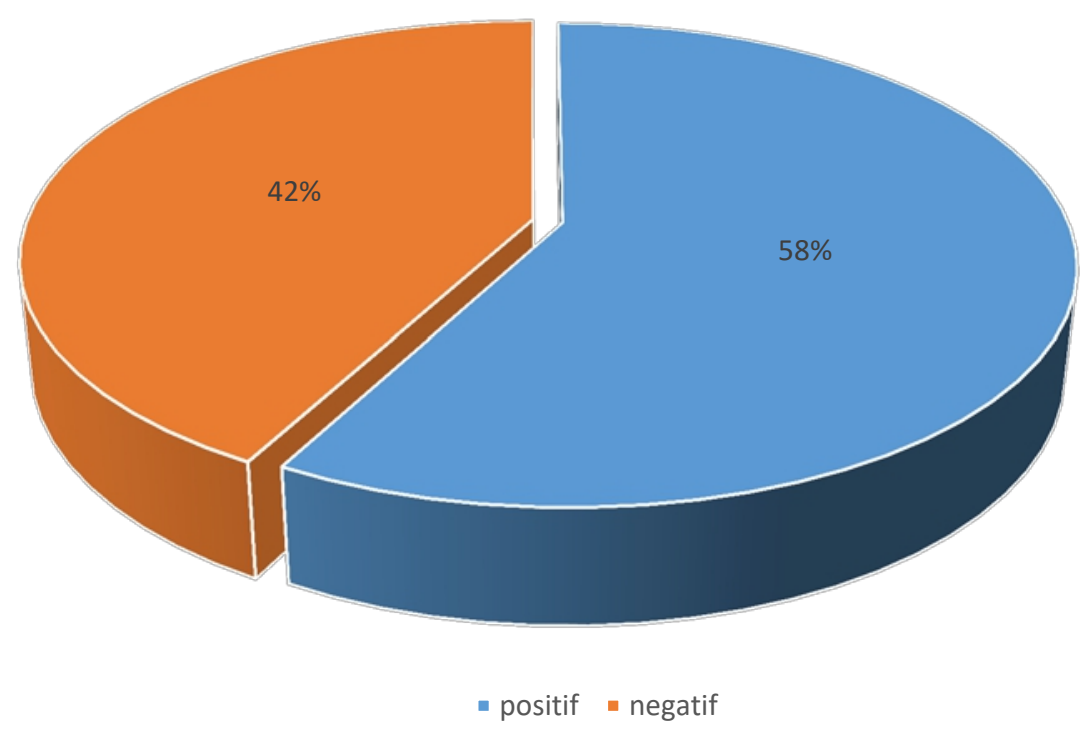

Figure 2: Taux de contamination des carcasses bovines par des souches E. coli

La contamination par les souches de $E$. coli était variable en fonction du site de prélèvement des échantillons (cou, cuisse, bras, flanc) sur les carcasses bovines. Ainsi, les échantillons collectés au niveau du bras étaient les plus contaminés (70\%) suivis de ceux collectés au niveau de la cuisse et du flanc (55\%) et enfin ceux prélevés au niveau du cou (51,7\%) (Figure 3). 


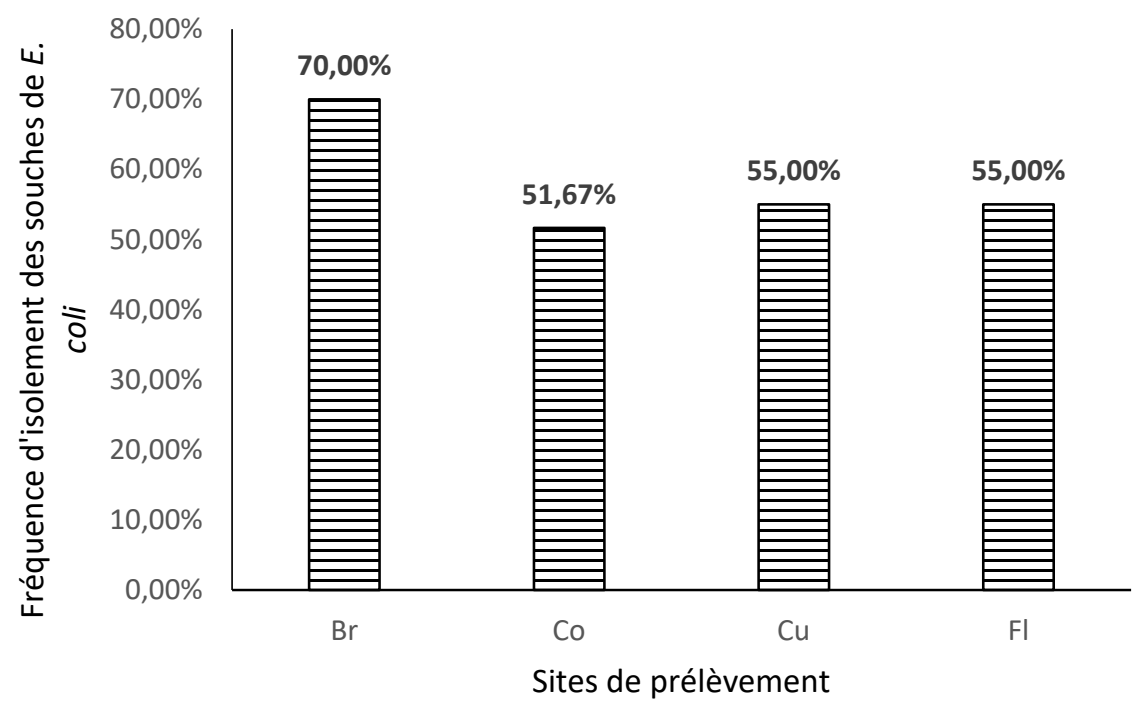

$\mathrm{Br}$ : Bras ; Co : cou ; Cu : Cuisse ; Fl : Flanc

Figure 3: Taux de contamination des échantillons de carcasses bovines en fonction des sites de prélèvement.

\section{Antibiorésistance des souches d' $E$.coli isolées des carcasses bovines}

La susceptibilité des 139 souches d'E. coli isolées des carcasses bovines était variable en fonction des 15 antibiotiques testés (Figure 4). En effet, les données revèlent que les souches d'E. coli développaient une forte résistance à la ceftriaxone $(88,49 \%)$ et les faibles taux de résistance ont été observés avec la gentamicine $(1,44 \%)$.

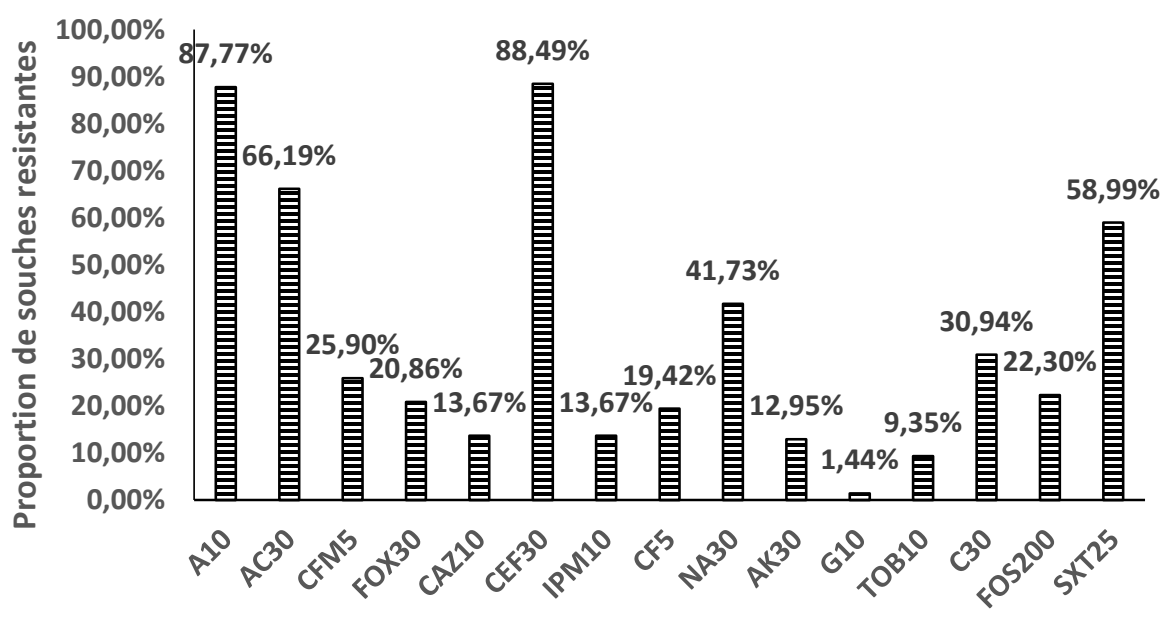

Antibiotiques testés

A10 : Ampicilline (10 $\mu \mathrm{g})$, AC30 : amoxicilline + acide clavuanique $(30 \mu \mathrm{g})$, CFM5 céfixime $(5 \mu \mathrm{g})$, FOX30 : céfoxitine $(30 \mu \mathrm{g})$, CAZ10 : ceftazidime $(10 \mu \mathrm{g})$, CEF30 : 
ceftriaxone (30 $\mu \mathrm{g})$, IMP10 : Imipénème $(10 \mu \mathrm{g})$, CF5 : ciprofloxacine $(5 \mu \mathrm{g}), \mathrm{NA30}$ : acide nalidixique (30 $\mu \mathrm{g})$, AK30 : amikacine (30 $\mu \mathrm{g}), \mathrm{G} 10$ : gentamicine $(10 \mu \mathrm{g})$, TOB10 :

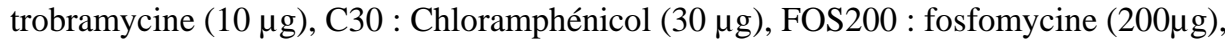
SXT25 : triméthoprime-sulfaméthoxazole (25 $\mu \mathrm{g})$.

Figure 4: Variabilité de la résistance aux antibiotiques des souches d'E. coli isolées des carcasses bovines

La résistance aux antibiotiques variait également en fonction des sites de provenance des souches (Figure 5).

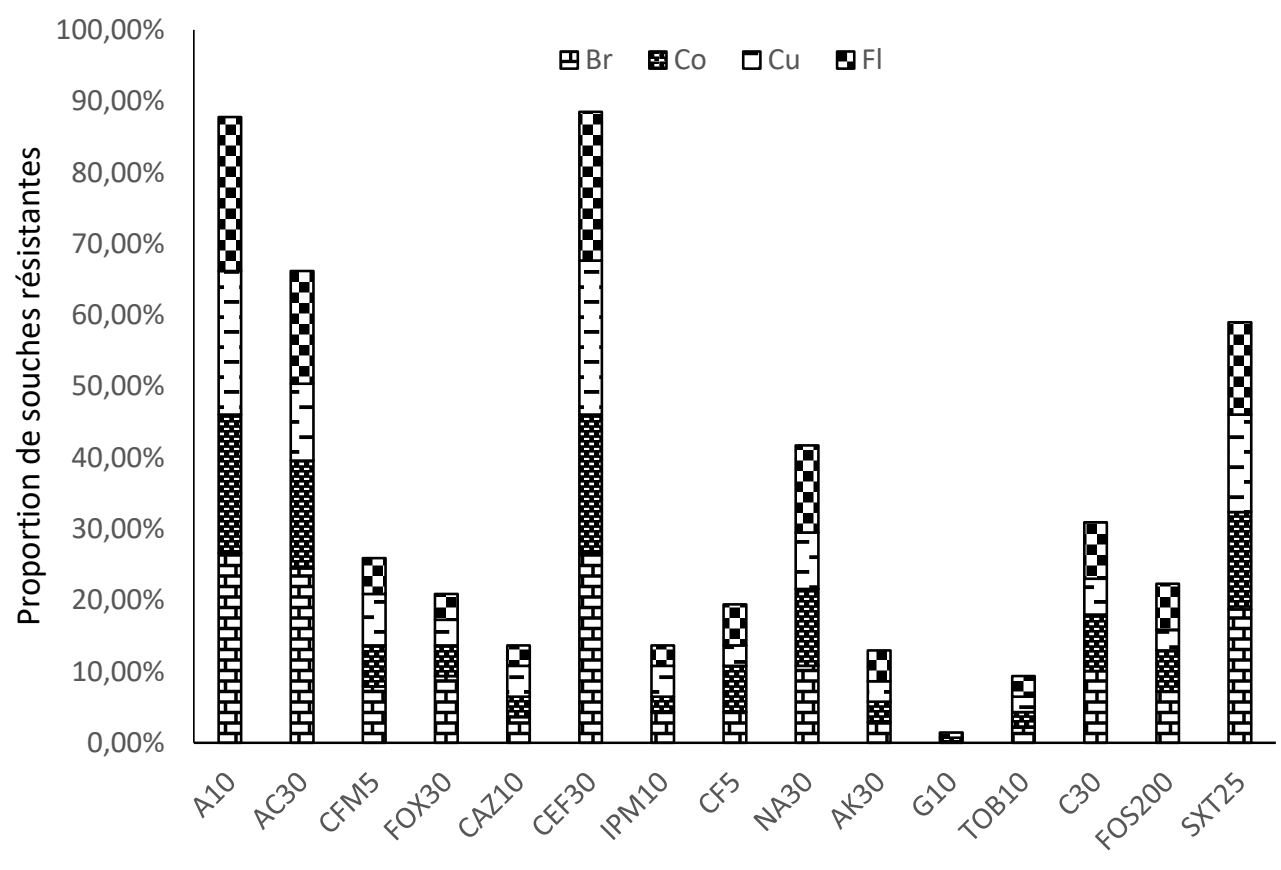

Antibiotiques testés

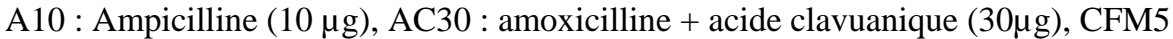
céfixime $(5 \mu \mathrm{g})$, FOX30 : céfoxitine $(30 \mu \mathrm{g})$, CAZ10 : ceftazidime $(10 \mu \mathrm{g}), \mathrm{CEF} 30$ : ceftriaxone $(30 \mu \mathrm{g})$, IMP10 : Imipénème $(10 \mu \mathrm{g})$, CF5 : ciprofloxacine $(5 \mu \mathrm{g})$, NA30 : acide nalidixique (30 $\mu \mathrm{g})$, AK30 : amikacine $(30 \mu \mathrm{g}), \mathrm{G} 10$ : gentamicine $(10 \mu \mathrm{g})$, TOB10 :

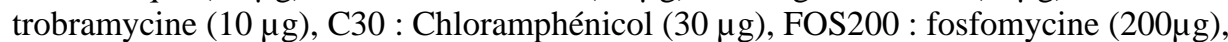
SXT25 : triméthoprime-sulfaméthoxazole $(25 \mu \mathrm{g})$.

Figure 5: Profil de résistance des souches d'E. coli aux antibiotiques en fonction des sites de collecte

Détection phénotypique des souches d'E. coli productrices de Pénicillinase et de BSLE

Parmi les 139 échantillons de E. coli isolés testés, 135 (97,12\%) ont été révélés positifs pour la production de pénicillinase. Une seule souche était productrice de BSLE. 


\section{Détection génotypique des $\boldsymbol{\beta}$-lactamases et de production de toxines}

Les souches de $E$. coli portent, à des proportions variables, les gènes de

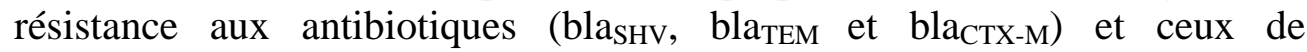
production de toxines (Véro toxine et entéro-toxine thermolabile). Ainsi, les données enregistrées révèlent que $12,82 \%$ des souches de $E$. coli étaient porteuses de gènes Véro toxine (VT), alors que 43,88\% avaient à la fois VT+ bla $_{\text {SHV }}$ Par contre aucune souche testée ne possédait le gène LT et bla $\mathrm{C}_{\mathrm{CTX}-\mathrm{M}}$ (Tableau 2).

Tableau 2: Répartition des gènes codant pour la résistance et pour la production de toxines portés par des souches d'E. coli isolées des carcasses bovines.

\begin{tabular}{lcc}
\hline Gènes & Nombre de souches porteurs & Pourcentage (\%) \\
\hline bla $_{\text {SHV }}$ & 21 & 26,92 \\
VT & 10 & 12,82 \\
VT et bla & 61 & 43,88 \\
bla $_{\text {Tem }}$ & 56 & 40,29 \\
bla $_{\text {CTX-M }}$ & 0 & 0 \\
LT & 0 & 0 \\
\hline
\end{tabular}

\section{Discussion}

La présente étude révèle globalement que 57,92\% des carcasses prélevées étaient contaminées par des souches de $E$. coli. Ce taux enregistré dans notre étude est largement supérieur à celui de 10\% observé en Australie (Phillips et al., 2001). Néanmoins, le taux enregistré dans notre étude se rapproche des $44 \%$ de carcasses de bovin contaminées par E. coli aux EtatsUnis (Siragusa et al., 1998). E. coli est un indicateur des bonnes pratiques de travail et des bonnes pratiques d'hygiène. Ainsi, la présence d'E. coli est également une indication de contamination fécale et de la présence potentielle d'agents pathogènes similaires du point de vue écologique. Cet indicateur est une mesure de l'implémentation des bonnes pratiques de travail, et de la prévention de la survenue et de la propagation d'une contamination fécale pendant le processus d'abattage et la découpe des viandes de bœuf. En ce qui concerne la contamination d'origine viscérale ou intestinale, Mackey et al. (1993) rappellent qu'elle est diminuée suite à l'arrêt de l'alimentation 6 à 8 heures avant le transport pour l'abattoir. De même, un bon état de santé, la mise à jeun et une tuerie effectuée dans le respect de l'hygiène limitent la contamination des viandes par les coliformes. Il est à noter que la contamination varie d'un site de prélèvement à un autre. Ainsi, en ce qui concerne $E$. coli, le bras se révèle être le site le plus contaminé (70,0\%) suivi de la cuisse et du flanc (55,00\%). Le cou est le site le moins contaminé par $E$. coli. Cette variabilité de contamination indique que les différentes parties de la carcasse bovine n’ont pas les mêmes taux d’exposition à la contamination microbienne. 
Les résultats de la susceptibilité aux antibiotiques des souches d'E. coli montrent que ces dernières présentaient une sensibilité variable vis-à-vis des quinze antibiotiques testés. Ainsi, 88,49\% des souches étaient résistantes à la ceftriaxone; $87,77 \%$ à l'ampicilline et $66,19 \%$ à l'amoxicilline + acide clavulanique. Ces proportions apparaissent inférieures aux 95,2\% rapportés par Anago et al. (2015) au Bénin. Cette différence peut s'expliquer par le fait que les souches étudiées par ces auteurs étaient les souches d'origine clinique, donc qui ont été en contact permanent avec les antibiotiques. De plus, les taux élevés de résistance (71\%), mais légèrement inférieurs aux nôtres à la ceftriaxone ont été rapportés par Mousse et al. (2016) au niveau des aliments de rues. Orden et al. (2000) ont examiné 195 souches de E. coli isolées chez des bovins diarrhéiques en Espagne et fait état d'une haute résistance à l'ampicilline, la tétracycline, et le triméthropime; ces résultats sont en accord avec ceux présentés dans ce travail (figure 3). La persistance de la résistance au chloramphénicol des souches de $E$. coli a été également observée par d'autres auteurs (Bischoff et al., 2005). Les forts taux observés dans le domaine alimentaire et en santé animale doivent être comme une sonnette d'alarme sur la manière d'utiliser les antibiotiques. En effet, les microorganismes résistants sont très facilement diffusés au sein d'unités par contact fécal, la contamination de l'eau utilisée par les animaux ou la contamination environnementale du sol (Teuber, 2001). Les bactéries résistantes aux antimicrobiens provenant d'animaux peuvent coloniser la population humaine à travers la chaîne alimentaire; c'est ainsi que ces bactéries résistantes peuvent être facilement transférées à l'homme par les animaux utilisés comme sources de nourriture (van den Bogaard et Stobberingh, 2000).

Les taux de résistance observés peuvent être des indicateurs de la probable provenance des microorganismes. Ainsi, les antibiotiques les plus actifs de notre étude étaient la gentamycine (98,56\%), la tobramycine (90,65\%), l'amikacine (87,05\%) et l'imipenème (86,33\%). Ces antibiotiques sont souvent les plus efficaces pour le traitement des infections à E. coli. Ceci suggère que les souches isolées pourraient provenir des manipulateurs des carcasses bovines au cours du processus.

Nous avons remarqué que $97,12 \%$ des souches produisaient la pénicillinase contre $0,7 \%$ des souches de $E$. coli isolées qui étaient productrices de ß-lactamases à spectre élargi (BLSE). Ce taux n'est pas très loin de $0,4 \%$ observé par Mesa et al. (2006) dans les aliments. Toutefois, ce taux est très différent de ceux rapportés dans le milieu hospitalier ; $16 \%$ au Cameroun (Lonchel et al., 2012) et 33,33\% en Amérique (Dexheimer et al., 2015). La présence des gènes codant pour bla $\mathrm{Tem}(40,29 \%)$ et bla SHV (26,92\%) confirme la résistance phénotypique des souches de $E$. coli aux ßlactamines. 
Dans la présente étude, 12,82\% des souches possèdent le gène codant pour la sécrétion des Véro toxines encore appelées Shiga toxines. Ceci dénote alors de la présence d'Escherichia coli entéro-hémorragiques dans les carcasses bovines. En effet, les bovins ont longtemps été considérés comme le principal réservoir de souches de STEC. Et dans ce réservoir, plus de 200 sérotypes de STEC différents ont été isolés, certains étant plus fréquemment isolés que d'autres (Beutin et al., 2004 ; Caprioli et al., 2005). Lira et al. (2004) ont rapporté l'isolement de souches de STEC à partir du lait de mammite, le transport du gène STX était de 12,08\%. Ce taux se rapproche des résultats rapportés dans la présente étude. Un grand nombre de d'Escherichia coli vérotoxinogènes sont associés à des infections intestinales humaines, et certains de ces sérotypes sont reconnus comme pathogènes d'origine alimentaire importants. Ces souches peuvent, de ce fait, causer une diarrhée aqueuse ou une colite hémorragique qui peut évoluer vers le syndrome hémolytique et urémique chez les jeunes enfants ou micro angiopathie thrombotique chez les adultes. La viande de bœuf crue ou hachée est donc une source majeure d'E. coli pathogènes et peut supposer que ces organismes, dans les matières fécales des bovins, sont transférés à la viande lors de l'abattage. Les bovins et leur environnement sont parmi les sources les plus importantes d'E. coli pathogènes, et ils peuvent être impliqués dans la transmission directe d'E. coli chez les humains.

\section{Conclusion:}

Les carcasses bovines provenant de l'abattoir Cotonou/Porto novo sont fortement contaminées par Escherichia coli. Ces bactéries sont potentiellement capables de causer les toxi- infections vu leur forte résistance aux antibiotiques et la capacité de produire des toxines. Pour ce faire, les conditions hygiéniques doivent être mises à jour par tous les acteurs intervenant le long de la chaine de transformation de la viande de bœuf. Néanmoins, il serait intéressant de dresser le profil génotypique de ces souches ainsi isolées.

\section{References:}

1. Allouch, P.Y., Labia, R., Pina, P., \& Morin, E. (1995). Observatoires hospitaliers de la sensibilité d'Escherichia coli et de Klebsiella pneumoniae à l'association Amoxicilline/Acide clavulanique en 1994. Médecines et Maladies Infectieuses, 25, (8-9) 934- 944. doi: 10.1016/S0399-077X(05)81150-3

2. Anago, E., Ayi-Fanou, L., Akpovi, C.D., Hounkpe, W.B., Tchibozo, M.A., Bankole, H.S., \& Sanni, A. (2015). Antibiotic resistance and genotype of beta-lactamase producing Escherichia coli in nosocomial 
infections in Cotonou, Benin. Annals of Clinical Microbiology and Antimicrobials, 14, 5. doi: 10.1186/s12941-014-0061-1

3. Aranda, S.H., Fabbricotti, U., Fagundes-Neto, U., \& Scaletsky, I.C.A. (2007). Single multiplex assay to identify simultaneously entropathogenic, enteroaggregative, enterotoxigenic, enteroinvasive and Shiga toxin-producing Escherichia coli strains in Brazilian children. FEMS Microbiology Letters, 264(2), 145-150.

4. Beutin, L., Krause, G., Zimmermann, S., Kaulfuss, S., \& Gleier, K. (2004). Characterization of Shiga toxin-producing Escherichia coli strains isolated from human patients in Germany over a 3-year period. Journal of Clinical Microbiology, 42, 1099-1108. doi: 10.1128/JCM.42.3.1099-1108.2004.

5. Bischoff, K.M., White, D.G., Hume, M.E., Poole, T.L., \& Nisbet, D.J. (2005). The chloramphenicol resistance gene cmlA is disseminated on transferable plasmids that confer multiple-drug resistance in swine Escherichia coli. FEMS Microbiology Letters, 243(1), 285-291.

6. Caprioli, A., Morabito, S., Brugere, H., \& Oswald, E. (2005). Enterohaemorrhagic Escherichia coli: emerging issues on virulence and modes of transmission. Veterinary Research, 36(3), 289-311.

7. Clifton-Hadley, F.A. (2000). Detection and diagnosis of Escherichia coli $\mathrm{O} 157$ and other verocytotoxigenic E. coli in animal faeces. Reviews in Medical Microbiology, 11(1), 47-58.

8. De Wals, P. (2007). Analyses mutationnelles et cinétiques de la $\beta$ lactamase TEM-1 de Escherichia coli. Vers une meilleure compréhension du phénomène de résistance aux antibiotiques. Mémoire en Biochimie. Faculté de médecine, Université de Montréal. Disponible à: https://papyrus.bib.umontreal.ca/xmlui/bitstream/handle/1866/15245/ De_Wals_PierreYves_2007_memoire.pdf?sequence=1\&isAllowed=y

9. Dexheimer, G.M., Prediger, J., Weidlich, L., \& Pozzobon, A. (2015). Prevalence of resistance and molecular characterization of extended spectrum beta-lactamase (ESBL) - producing bacteria isolated in a hospital in Southern Brazil. African Journal of Microbiology Research, 9(5), 294-300. Doi: 10.5897/AJMR2014.7340

10. El-Hadef El Okki, S., El-Groud, R., Kenana, H., \& Quessy, S. (2005). Evaluation de la contamination superficielle des carcasses bovines et ovines provenant de l'abattoir municipal de Constantine en Algérie. Canadian Veterinary Journal, 46 (6), 638-640.

11. Gangoué-Piéboji, J., Bedenic, B., Koulla-Shiro S., Randegger, C., Adiogo, D., Ngassam, P., Ndumbe, P., \& Hächler, H. (2005). 
Extented-spectrum- $\beta$-lactamase-producting Enterobacteriaceae in Yaounde, Cameroon. Journal of Clinical Microbiology, 43(7), 32733277.

12. Gassama-Sow, A. Sow, P.S., Guèye, M., Guèye-N'diaye, A., Perret, J.L., M'boup, S., \& Aïdara-Kane, A. (2004). Characterization of pathogenic Escherichia coli in human immunodeficiency virusrelated diarrhea in Senegal. Journal of Infectious Diseases, 189(1), 75-78.

13. Jarlier, V., Nicolas, M., Fournier, G., \& Philippon, A. (1988). Extended broad-spectrum $\beta$-lactamases conferring transferable resistance to newer $\beta$-lactam agents in Enterobacteriaceae: hospital prevalence and susceptibility patterns. Review Infection Diseases, 10, 867-878.

14. Johnson, J.R., \& Russo, T.A. (2005). Molecular epidemiology of extraintestinal pathogenic (uropathogenic) E. coli. International Journal of Medical Microbiology, 295(6-7), 383-404.

15. Kaper, J.B., Nataro, J.P., \& Mobley, H.L. (2004). Pathogenic Escherichia coli. Nature Reviews Microbiology, 2(2), 123-40. doi:10.1038/nrmicro818.

16. Lira, W.M., Macedo, C., \&Marin, J.M. (2004). The incidence of Shiga toxin-producing Escherichia coli in cattle with mastitis in Brazil. Journal of Applied Microbiology, 97(4), 861-866.

17. Lonchel, C.M., Meex, C., Gangoué-Piéboji, J., Boreux, R., Assoumou, M.C., Melin, P., \& De Mol, P. (2012). Proportion of extended-spectrum ß-lactamase producing Enterobacteriaceae in community setting in Ngaoundere, Cameroon. BMC Infectious Diseases, 12, 53. doi: 10.1186/1471-2334-12-53.

18. Mackey, B.M., Miles, C.A., Seymour, D.A., \& Parsons, S.E. (1993). Thermal denaturation and loss of viability in Escherichia coli and Bacillus stearothermophilus. Letters in Applied Microbiology, 16, 56-58. doi:10.1111/j.1472-765X.1993.tb00341.x

19. Mesa, R.J., Blanc, V., Blanch, A.R., Cortés, P., González, J.J., \& Lavilla, S. (2006). Extended-spectrum $\beta$-lactamase-producing Enterobacteriaceae in different environments (humans, food, animal farms and sewage). Journal of Antimicrobial Chemotherapy, 58(1), 211-215

20. Moussé, W., Baba-Moussa, F., Adjanohoun, A., Noumavo, P.A., Sina, H., Assogba, S., \& Baba-Moussa, L. (2016). Virulence profiles of pathogenic Escherichia coli strains isolated from street foods in Benin. International Journal of Biotechnology and Food Science, 4(3), 52-64. 
21. Nüesch-Inderninen, M.T., Hachler, H., \& Kayser, F.H. (1996). Detection of genes coding for extended-spectrum SHV betalactamases in clinical isolates by a molecular genetic method, and comparison with the $\mathrm{E}$ test. European Journal of Clinical Microbiology and Infectious Diseases, 15(5), 398-402.

22. Orden, J.A., Ruiz-Santa-Quiteria, J.A., Garcia, S., Cid, D., \& De La Fuente, R. (2000). In vitro susceptibility of Escherichia coli strains isolated from diarrhoeic dairy calves to 15 antimicrobial agents. Journal of Veterinary Medicine, 47(5) 329-335.

23. Phillips, D., Sumner, J., Alexander, J.F., Dutton, K.M. (2001). Microbiological quality of Australian sheep meat. Journal of Food Protection, 5, 697-700.

24. Siragusa, G.R., Dorsa, W.J., Cutter, C.N., Bennett, G.L., Keen, J.E., \& Koohmaraie, M. (1998). The incidence of Escherichia coli on beef carcasses and its association with aerobic mesophilic plate count categories during the slaughter process. Journal of Food Protection, 61(10), 1269-1274.

25. SFM (Société Française de Microbiologie), (2015). Recommandations 2015 du Comité de l'Antibiogramme de la Société Française de Microbiologie, disponible sur le site: http://www.sfmmicrobiologie.org/UserFiles /files/casfm/CASFM_EUCAST_V1_2015.pdf

26. Teuber, M. (2001). Veterinary use and antibiotic resistance. Current Opinion in Microbiology, 4(5), 493-499.

27. Thomson, K.S., \& Sanders, C.C. (1992). Detection of extendedspectrum $\beta$-lactamases in members of the family Enterobacteriaceae: comparison of the double-disk and three-dimensional tests. Antimicrobial Agents Chemotherapy, 36(9), 1877-1882.

28. van den Bogaard, A.E., \& Stobberingh, E. (2000). Epidemiology of resistance to antibiotics links between animals and humans. International Journal of Antimicrobial Agents, 14(4), 327-335.

29. WHO (World Health Organization) (2015). WHO Estimates of the Global Burden of Foodborne Diseases; Foodborne disease Burden epidemiology reference group 2007 - 2015. Disponible sur le lien:

30. http://apps.who.int/iris/bitstream/10665/199350/1/9789241565165_e ng.pdf?ua=1 